Review Article

\title{
NOVEL NANOCARRIERS FOR ETHNOPHARMACOLOGICAL FORMULATIONS
}

\author{
V. SIVAPRIYA, S. PONNARMADHA, N. ABDUL AZEEZAND, V. SUDARSHANADEEPA*
}

Nano-bio Translational Research Laboratory, Department of Biotechnology, Bannari Amman Institute of Technology, Sathyamangalam 638401, Erode District, Tamil Nadu

Email: sudarshanadeepav@bitsathy.ac.in

Received: 20 Mar 2018, Revised and Accepted: 24 May 2018

\section{ABSTRACT}

A numerous novel drug delivery system has been emerged by combining herbal medicine with nanotechnology to administer drugs encompassing the enhancement of compatibility and efficacy. The herbal phytoconstituents are compatible compared to the chemical active pharmaceutical ingredients (APIs). But the therapeutic consequence of the phytoconstituent is limited due to poor aqueous solubility. Therefore, the demand to develop a system which improves the solubility of the phytomedicine is mounting rapidly. Nanotechnology plays a vital role in increasing the solubility, enhancing the bioavailability and improving the drug specificity of bioactive constituents. Nanosystems such as liposomes, nanoparticles, phytosomes, ethosomes, nanoemulsions and solid lipid nanoparticles are used to deliver the bioactive constituent at an adequate dose to the site of action and during the entire treatment period. The current review discusses the various novel drug delivery systems which have been developed to attain better therapeutic response of the herbal drug.

Keywords: Bioavailability, Nanocarriers, Drug delivery, Ethnopharmacology, Phospholipid bilayer, Vesicular drug delivery

(C) 2018 The Authors. Published by Innovare Academic Sciences Pvt Ltd. This is an open access article under the CC BY license (http://creativecommons.org/licenses/by/4.0/) DOI: http://dx.doi.org/10.22159/ijap.2018v10i4.26081

\section{INTRODUCTION}

Plants have long been recognized for their therapeutic properties. For centuries, aboriginal cultures around the world have used traditional herbal medicine to treat a myriad of diseases [1]. The demand for herbal medicines has surged recently due to its ability to treat diseases with negligible or lesser side effects [2]. There has been a chief concern using modern medicines in treating diseases like cancer using chemotherapy, hormone-blocking therapy, monoclonal antibodies, and their kinds. They create the aftermaths and adverse effects in addition to the therapeutic complications and cost. Ethnopharmacological medicines are an ideal alternate to these concerns.

Some major water-insoluble phytoconstituents like flavonoids and tannins suffer from confines like poor water solubility, lipophilicity and inappropriate molecular size consequential in poor absorption and bioavailability [3]. But the technique by which a drug is transported can have a substantial consequence on its efficacy [4]. Hence, the research across the globe has begun to ponder on formulating the delivery system without having to change the nature of the phytoconstituents of the herbs.

Various drug delivery systems and drug targeting approaches are now under progress to curtail drug deprivation, prevent destructive sideeffects and increase drug bioavailability $[5,6]$. Some of the novel drug delivery approaches are effectual in improving the dissolution properties of poorly water-soluble drugs in accomplishing controlled drug delivery systems which delivers the drug at a rate dictated by the need of the body over a period of treatment at the site of action. Carrier-mediated drug delivery systems can be applied for herbal drugs to safe, effective and time-tested products [7]. The carriers can be made gradually degradable, prompt reactive and even targeted. Different types of nanomaterials are used as nanocarriers which permit hydrophobic and hydrophilic drugs to deliver throughout the body which can be either dissolved, entrapped or encapsulated to the polymer matrix to intensify the solubility, sustained release effects, can be protected from degradation and can even be targeted to the site of action $[8,9]$. Therefore, various types of nanocarriers can be made up of natural and synthetic polymers, polysaccharides and lipids such as polymeric nanoparticles, solid lipid nanoparticles, liposomes and phytosomes for better therapeutic responses by integrating with traditional medicine system (fig. 1). These types of novel drug delivery systems enhance the solubility, bioavailability, stability, pharmacological activity of the drug molecule and also protect it from physical and chemical degradation.

\section{Search criteria}

The sources and range of years used to write this review articles are Pubmed, Google scholar, Science direct and 2014-2018 respectively.

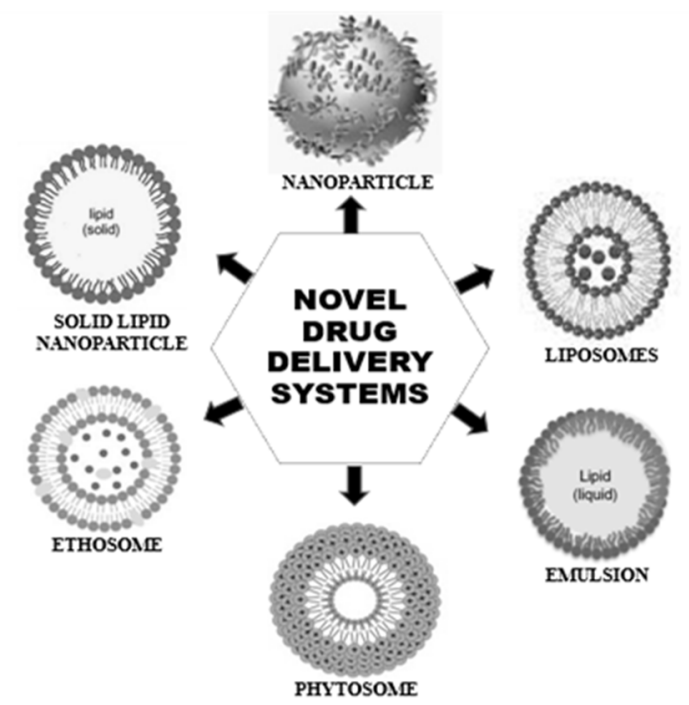

Fig. 1: An overview of different drug carriers [4]

Drug delivery systems are based on interdisciplinary approach, that slowly delivers drug at a controlled rate. They amend drug release profile, absorption, distribution and elimination thereby improving the product efficacy as well as the pharmacokinetic and pharmacodynamic characteristics of various drug molecules to potentially overcome the limitations of conventional therapeutics [10].

\section{Nanoparticles}

Nanoparticles are colloidal systems whose particle size ranges from 10 to $100 \mathrm{~nm}$ [11]. It has numerous advantages which includes enhanced solubility and bioavailability, size reduction and better absorption of herbal medicine [12]. This type of drug delivery system should be efficient enough to circulate in the bloodstream and to target cells and 
tissues. They are also capable of releasing biologically active constituents to the site of action at a therapeutically optimal rate in a dose-dependent manner [13]. There are different biological and nonbiological methods of nanoparticle synthesis which is economical, ecofriendly and also cost-effective [14-16].

Raksha et al. formulated paclitaxel loaded nanoparticulate system which appears to be capable in carrying as well as targeted delivery of paclitaxel with enhanced therapeutic efficacy and safety thereby minimizing the adverse effects [17]. Khdair et al. developed novel chitosan-modified polymeric nanoparticles by double emulsion solvent evaporation technique which proficiently encapsulated anticancer drugs, and sustained the release, enhanced oral bioavailability, and improved tumour cellular uptake of the drugs [18]. Patricia et al. developed three different lignin nanoparticles which show better stability, biocompatibility, uniform dispersity, ability to load poorly soluble drugs, good cellular interaction and sustained drug release for disease diagnosis and therapy [19]. Jagminas et al. reported a novel method to synthesise methionine mediated super paramagnetic Cobalt ferrite nanoparticle linked with gold quantum dots which has promising application in targeted drug delivery [20]. Chen et al. inspected a pH-sensitive drug release system for targeting CD44 receptor using doxorubicin conjugated mesoporous silica nanoparticles which exhibited improved drug effectiveness and better stability at functional $\mathrm{pH}$ for novel preparation of drug delivery systems [21]. Villeret et al. Silver nanoparticles act as potential antivirals for treating human infectious disease i. e specifically influenza virus infection. It enhanced chemokine (C-C motif) 5 and interferon beta cytokines that might have practical implication in clinical application [22].

\section{Liposomes}

Liposomes are lipid bilayers of 50-1000 $\mathrm{nm}$ in diameter that act as convenient vehicles to deliver biologically active agents [23]. They are usually made up of phospholipids which can incorporate both hydrophilic and lipophilic molecules [24, 25]. They improve drug solubility, bioavailability and stability. They can even combine with site-specific ligands to attain active targeting. They also enhance the therapeutic activity and safety of drugs chiefly by delivering them at the site of action and by sustaining the therapeutic levels of drug for a prolonged period of time [26, 27].

Hadis et al. reported that the liposomes are one of the most advanced and reliable techniques for the progress of useful bioproducts particularly in medical diagnostics [28]. Maussang et al. formulated PEGylated liposomes conjugate with glutathione for safely deliver the encapsulated drugs to the brain which serve as promising drug delivery technology for the treatment of CNS disorders [29]. Xiao et al. developed sorafenib and gadolinium coloaded liposomes to improve the sparsely water soluble sorafenib and to screen its distribution and the early response on its in vivo treatment in a precise manner and found that this could be a promising nano-carrier for the MRI-guided in vivo visualization of the delivery and Hepatocellular carcinoma treatment [30]. Sharma et al. proposed an advanced approach to target C-typed lectin receptor through nanoliposome by mannose surface modification for active targeting which is a promising system for effective cancer immunotherapy [31]. Abraham et al. prepared a safe and efficient nanoliposome for the proper delivery of therapeutic mRNA for treating various diseases such as cancer, inflammatory as well as cardiovascular diseases [32]. Ju et al. studied octreotide altered daunorubicin conjugated liposomes loaded with dihydroartemisinic that showed improved cellular uptake and cytotoxicity while evaluated on breast cancer cells and xenografts nude mice models [33]. Tatodeet al. used response surface methodology to formulate paclitaxel-loaded liposomes by thin film hydration method which demonstrates better enhancement of in-vitro drug release [34].

\section{Nanoemulsions}

Nanoemulsions are also called as oil-in-water emulsions composed of oil droplets and stabilized by surfactants [35, 36]. The average size of the oil droplet in nanoemulsion is between 100 and $500 \mathrm{~nm}$. It is prepared by mixing oil phase with an aqueous phase under high mechanical extrusion process $[37,38]$. Both hydrophilic and lipophilic molecules can be efficiently incorporated. This is one of the efficient systems for enhancing drug delivery.

Zheng et al. integrated nanoemulsions into hydrogels which shows great potential for therapeutic purposes through topical or systemic administration. They also claimed that the prepared nanoemulsion is more stable and safe [39]. Mou et al. developed nanoemulsion gelbased topical delivery of Amphotericin which is an economic approach for enhanced and sustained permeation of drug against fungal assessment [40]. Primo et al. denominated magnetic nanoemulsion which enhances the diffusion of the drug when associated with Foscanfor the application of photodynamic therapy [41]. Mahato et al. reported that nanoemulsionsserve as the effective drug delivery as a carrier of nucleic acids, drugs as well as imaging agent which has been widely used for disease diagnostics, imaging and therapy [42]. Jasmina et al. prepared nanoemulsions by both higher energy and lower energy emulsion methods and concluded that low energy methods are greatly recognized by the manufacturers as they do not require any expensive equipment [43]. $\mathrm{Wu}$ et al. determined the absorption mechanism ofBaicalin mediated nanoemulsion by in situ single-pass intestine perfusion (SPIP) method and chylomicron-blocked rat model for effectual oral delivery [44]. Gueutin et al. evaluated erythromycin loaded nanoemulsion by hot high-pressure homogenization method which increases the stability of erythromycin in an acidic condition as it acts as a suitable system for Helicobacter pylori treatment [45].

\section{Phytosome}

Phytosome is also known as phytolipids delivery system which forms a bridge between the conventional drug delivery system and novel drug delivery system. The polyphenolic phytoconstituents are complexed with phospholipid to produce lipid compatible molecular complexes in the equimolar ratio [46]. They improve the absorption of poorly water-soluble drugs through various routes of administration thereby increasing the bioavailability and therapeutic index when compared to traditional drug delivery systems $[47,48]$. They also enhance the capacity of drugs to cross the lipid bilayer thereby reaching the systemic circulation [49].

Chetan et al. reported that phytosome shows improved pharmacokinetic and pharmacodynamic effect than conventional botanical extracts which can be utilized for treating various diseases [50]. The prepared phytosome using sinigrin shows higher efficiency than control to release the compound in a slow and controlled manner into the stratum corneum for wound healing [51]. Abdelkader et al. formulated L-carnosine phytosome by combining hydrogel and lipid-based carrier which shows sustained drug pervasion and inhibits the browning of the lens and protects from cataract formation [52]. Damle et al. focused on encapsulating orange peel and liquorice extracts into phospholipid complex which exhibited as a better topical dosage delivery towards skin that ensured high cutaneous absorption of phytoconstituents in the skin for long period of action in contradiction to skin ageing [53]. Das et $a l$. proposed the synthesized rutin-phytosome complex increases the transdermal absorption rate to treat various diseases such as arthritis, rheumatism, etc. with controlled and sustained drug release [54]. The lyophilized technique was found to be more efficient to deliver diosmin when compared to other methods (solvent evaporation, salting out) of phytosomal preparation as it enhances the absorption and permeation of the drugs across the lipid bilayer as described by May et al. [55]. Telange et al. conjugated apigenin, a low aqueous soluble compound with phospholipid which act as an excellent potential to improve the in vivo antioxidant activity, solubility, bioavailability and pharmacological properties compared to free apigenin [56].

\section{Ethosomes}

Ethosomesare non-invasive lipid vesicular carrier system in which it composed of phospholipid increased the concentration of alcohol (mostly ethanol) and water [57]. High concentration of alcohol makes this system more unique. They effectively incorporate the delivery of lipophilic, hydrophilic and amphiphilic molecules to the body [58]. They promote high entrapment efficiency, better stability and controlled release of drugs through transdermal route to the 
systemic circulation [59]. They tend to be more superior than liposome as it delivers drug through the skin in terms of quantity and level of penetration [60].

Sharma et al. developed phospholipid-based carrier systems for the effective delivery of Aceclofenac and found that ethosome prepared by cold method offers high vesicle density and drug loading efficiency as compared with elastic membrane vesicles [61]. Shelke et al. optimized Zolmitriptan loaded ethosomal intranasal gel by three-level factorial design which could aid as an effective therapy for the recurrence of a migraine with controlled release and permeability [62]. Sujatha et al. observed the release of fiasterideethosome was first order kinetics with higuchi diffusion mechanism which plays a promising role in transdermal drug delivery [63]. Jain et al. formulated the ethosome loaded with Carbopol as hydrogel and evaluated the in vitro permeation studies using rat skin which facilitate effective carrier for the transdermal route of drug administration [64]. Ahmed et al. aimed to optimize the ethosomal formulation made up of glimepiride and integrating them into transdermal films can reduce side effects and prolong the drug release [65]. Shaik et al. explored Aceclofenac loaded ethosomeincreases the transdermal flux and serve as an effectual vehicle for the sustained release of the drug. They also optimized the ethanol and lecithin concentration to improve the skin permeability and concluded that increased ethanol enhances the drug diffusion [66]. Milind et al. developed ethosome for topical delivery of rizatriptan benzoate as site-specific approach and optimized for maximum entrapment efficiency [67].

\section{Solid lipid nanoparticles}

Solid lipid nanoparticles are spherical lipid particles remains in a solid state at room temperature [68]. Their size ranges from 50-1000 nm and are dispersed in aqueous surfactant solution [69]. Lipids used in the preparation of solid lipid nanoparticle can be stabilized by adding a biocompatible surfactant in the proper ratio [70]. They are generally made up of the hydrophobic core to which a monolayer of phospholipid is coated [71]. They have a tendency to carry both the hydrophilic and hydrophobic drugs for therapeutic purposes.

Kotmakç et al. synthesised small solid lipid nanoparticles below 100 $\mathrm{nm}$ by modified microemulsion method which exhibits lower toxicity on L929 cells for effective drug delivery [72]. Bhupinder et al. developed Acyclovir Solid Lipid Nanoparticles by high-pressure hot-homogenization technique were found to be the average size in nano range. It exhibited better entrapment efficiency and drug loading capacity in drug distribution pattern [68]. Thakkar et al. combined aspirin and curcumin with free sulforaphane entrapped chitosan solid lipid nanoparticle indicates the prolonged exposure of the developed formulation that act as effective therapy to prevent pancreatic cancer [73]. Chantaburananet al. developed Ibuprofenloaded solid lipid nanoparticle composed of different ratios of Softisan 378 and Cetyl palmitate which were found to have sustained release, decreased in particle size and surface charge of the particle for improved dermal drug delivery [74]. Wang et al. prepared ultra-fine powder using polysaccharide coated solid lipid nanoparticles and nanostructured lipid carrier by nanospray drying technology which can tend to produce uniform spherical lipid particle for improved lipid delivery system [75]. Mosallaeiet al. formulated solid lipid nanoparticle and PEGylated solid lipid nanoparticle containing 7-Ethyl-10-hydroxycamptothecin, an active component of irinotecan shows improved activity to hinder the occurrence of a tumour [76]. Chetoni et al. determined tobramycin as ion-pair combined with solid lipid nanoparticles for the treatment of ocular infections which favour the drug activity to effectively reach the inner parts of the eye [77]. The pharmacokinetic and biodistribution analysis of thymoquinone loaded solid lipid nanoparticle exposed that the formulation capably targets the brain and delivers the drug in a controlled manner to the target organ. It shows that Bioavailability and plasma half-life of TQ-SLNs significantly increased than TQ suspensions [78].

\section{CONCLUSION}

Nanotechnology has been recognized for natural bioactive compounds to safely transport drugs into systemic circulation through various novel drug delivery systems. They have a tendency to exploit various biological compounds which have poor solubility, permeability and bioavailability. Hence, novel drug delivery systems can be used to improve the potent of biological compounds thereby avoiding the first pass effect, dose frequency and side effects at low cost. Thus, the progress of nanotechnology clutches the great potential to improve the therapeutic approach in the development of novel drug delivery system.

\section{FINANCIAL SUPPORT}

Nil

\section{AUTHORS CONTRIBUTIONS}

All the author has contributed equally

\section{CONFLICT OF INTERESTS}

Declared none

\section{REFERENCES}

1. Li FS, Weng JK. Demystifying traditional herbal medicine with a modern approach. Nature Plants 2017;3:17109.

2. Priyanka P, Brahmeshwar M. Systematic review on interaction studies of synthetic antidiabetic drugs and herbal therapies. J Pharma Res 2017;16:86.

3. Tanmoy S, Buddhadev L, Swayam P. Incorporation of lipolysis in monolayer permeability studies of lipid-based oral drug delivery systems. Drug Delivery Transl Res 2017;17:1-12.

4. Nalini T, Sugantha Kumari V, Khaleel Basha S. Novel nanosystems for herbal drug delivery. World J Pharm Pharm Sci 2017;6:1447-63.

5. Kusum D, Nimisha J, Kusum SV. Importance of novel drug delivery systems in herbal medicines. Pharmacogn Rev 2010;4:27-31.

6. Fred L, Holger MR. Novel drug delivery systems tailored for improved administration of glucocorticoids. Int $\mathrm{J}$ Mol Sci 2017;18:1836.

7. Shweta P, Niraj G, Manish J. Carrier-mediated target drug delivery systems: a novel approach-(An overview). Int J Pharm Pract 2013;4:701-9.

8. Zhang L, Radovic-Moreno AF, Alexis F, Gu FX, Basto PA, Bagalkot $\mathrm{V}$, et al. Co-delivery of hydrophobic and hydrophilic drugs from nanoparticle-aptamer bioconjugates. Chem Med Chem 2007;2:1268-71.

9. Zhang G, Chan W, Langer F. Nanoparticles in medicine: therapeutic applications and developments. Clin Pharmacol Ther 2008;5:761-9.

10. Sarika P, Ashutosh B, Deepak S. Sustained release matrix technology and recent advance in the matrix drug delivery system: a review. Int J Drug Res Technol 2017;3:8.

11. Dan G, Guoxin X, Jianbin Luo. Mechanical properties of nanoparticles: basics and applications. J Phys D 2013;47:13.

12. Wengang Li, Xiuhua Z, Xiaoli S, Yuangang Zu, Ying L, Yunlong GE. Evaluation of antioxidant ability in vitro and bioavailability of trans-cinnamic acid nanoparticle by liquid antisolvent precipitate. J Nanomater 2016;29:16.

13. Akanksha Raj, Prasanna Shah, Namita Agrawa. Dose-dependent effect of silver nanoparticles (AgNPs) on fertility and survival of Drosophila: an in vivo study. J Nanomater 2017;5:124-31.

14. Kiranmai M. Biological and non-biological synthesis of metallic nanoparticles: scope for current pharmaceutical research. Indian J Pharm Sci 2017;79:501-12.

15. Pantidos N, Horsfall LE. Biological synthesis of metallic nanoparticles by bacteria, fungi and plants. J Nanomed Nanotechnol 2014;5:2157-79.

16. Priyanka S, Yu-Jin K, Dabing Z, Deok-Chun Y. Biological synthesis of nanoparticles from plants and microorganisms. Trends Biotechnol 2016;34:588-99.

17. Raksha G, Rakesh Kumar T, Anil Kumar M, Krishna C, Narendra Kumar J. Luteinizing hormone-releasing hormone peptide tethered nanoparticulate system for the enhanced antitumoral efficacy of paclitaxel. Nanomed 2016;7:588-99.

18. Khdair A, Hamad I, Alkhatib H, Bustanji Y, Mohammad M, Tayem R, et al. Modified-chitosan nanoparticles: Novel drug delivery systems improve oral bioavailability of doxorubicin. Eur J Pharm Sci 2016;93:38-44. 
19. Patricia F, Kalle Lintinen AK, Ville H, Zehua L, Tomas BR, Antti $\mathrm{R}$, et al. In vitro evaluation of biodegradable lignin-based nanoparticles for drug delivery and enhanced antiproliferation effect in cancer cells. Biomat 2017;121:97-108.

20. Jagminas A. Methionine-mediated synthesis of magnetic nanoparticles and functionalization with gold quantum dots for theranostic applications. J Nanotechnol 2017;8:1734-41.

21. Chen C, Sun W, Wang X, Wang Y, Wang P. pH-responsive nanoreservoirs based on hyaluronic acid end-capped mesoporous silica nanoparticles for targeted drug delivery. Int J Biol Macromol 2018;17:33559-66.

22. Villeret B, Dieu A, Straube M, Solhonne B, Miklavc P, Hamadi $\mathrm{S}$, et al. Silver nanoparticles impair retinoic acid-inducible gene i mediated mitochondrial anti-viral immunity by blocking the autophagic flux in lung epithelial cells. ACS Nano 2018;2:1-49.

23. Lisa S, Tejaswi V, Fatemeh M, Sherry YW, Anil KS, Susan H. Advances and challenges of liposome assisted drug delivery. Front Pharmacol 2015;6:286-92.

24. Yoshihiro T, Kaoru N, Yurina I, Fumie H. Enhancement of skin penetration of hydrophilic and lipophilic compounds by phsensitive liposomes. J Pharm Pharm Sci 2015;18:249-57.

25. Maria C, Nikolaos N, Natassa P. The significance of drug-to-lipid ratio to the development of optimized liposomal formulation. J Liposome Res 2017;18:1-36.

26. Ravi TPU, AbulKalam AM. Sustained release of green tea polyphenols from liposomal nanoparticles; release kinetics and mathematical modelling. Iranian J Biotech 2017;15:1322-41.

27. Durgavati Y, Kumar S, Deepak P, Ranu Kumari D. Liposomes for drug delivery. J Biotechnol Biomater 2017;7:4.

28. Hadis D, Ali E, Mohammad K, Samira A, Abolfazl A. Application of liposomes in medicine and drug delivery. Artif Cells Nanomed Biotechnol 2016;44:381-91.

29. Maussang D, Rip J, Kregten J, Den Heuvel A, Der Pol S, Der Boom B, et al. Glutathione conjugation dose-dependently increases brain-specific liposomal drug delivery in vitro and in vivo. Drug Discovery Today: Technol 2016;20:59-69.

30. Xiao Y, Liu Y, Yang S, Zhang B, Wang T, Jiang D, et al. Sorafenib and gadolinium co-loaded liposomes for drug delivery and MRI-guided HCC treatment. Colloids Surf 2016;141:83-92.

31. Sharma R, Mody N, Kushwah V, Jain S, Vyas SP. C-type lectin receptor(s)-targeted nanoliposomes: an intelligent approach for effective cancer immunotherapy. Nanomed 2017;16:1945-59.

32. Abraham MK, Peter K, Michel T, Wendel HP, Krajewski S, Wang X. Nanoliposomes for safe and efficient therapeutic mRNA delivery: a step toward nanotheranostics in inflammatory and cardiovascular diseases as well as cancer. Nanotheranostics 2017;2:154-65.

33. Ju RJ, Cheng L, Peng XM, Wang T, Li CQ, Song XL, et al. Octreotide-modified liposomes containing daunorubicin and dihydroartemisinin for treatment of invasive breast cancer. Artif Cells Nanomed Biotechnol 2018;30:1-13.

34. Tatode A, Patil A, Umekar M. Application of response surface methodology in the optimization of paclitaxel liposomes prepared by thin film hydration technique. Int J Appl Pharm 2018;10:62-9.

35. Hasani F, Pezeshki A, Hamishehkar H. Effect of surfactant and oil type on size droplets of betacarotene-bearing nanoemulsions. Int J Curr Microbiol Appl Sci 2015;4:146-55.

36. Wulff-Perez MA, Torcello-Gomez MJ, Galvez-Ruiz MartinRodriguez. Stability of emulsions for parenteral feeding: Preparation and characterization of $\mathrm{o} / \mathrm{w}$ nanoemulsions with natural oils and Pluronic f68 as a surfactant. Food Hydrocoll 2009;23:1096-102.

37. Lovelyn C, Anthony AA. Nanoemulsion, mechanical stress current state of nanoemulsions in drug delivery. J Biomater Nanobiotechnol 2011;2:626-39.

38. Parthasarathi S, Muthukumar SP, Anandharamakrishnan C. The influence of droplet size on the stability, in vivo digestion, and oral bioavailability of vitamin E emulsions. Food Funct 2016;7:2294-302.

39. Zheng Y, Ouyang WQ, Wei YP, Syed SF, Hao CS, Wang BZ, et al. Effects of carbopol® 934 proportion on nanoemulsion gel for topical and transdermal drug delivery: a skin permeation study. Int J Nanomed 2016;11:5971-87.

40. Mou D, Chen H, Du D, Mao C, Wan J, Xu H, et al. Hydrogelthickened nanoemulsion system for topical delivery of lipophilic drugs. Int J Pharm 2008;353:270-6.
41. Primo FL, Michieleto L, Rodrigues MA, Macaroff PP, Morais PC, Lacava ZG, et al. Magnetic nanoemulsions as drug delivery system for Foscan $®$ : Skin permeation and retention in vitro assays for topical application in photodynamic therapy (PDT) of skin cancer. J Magn Magn Mater 2007;311:354-7.

42. Mahato R. Nanoemulsion as targeted drug delivery system for cancer therapeutics. J Pharm Sci Pharmacol 2017;3:83-97.

43. Jasmina H, Dzana O, Alisa E, Edina V, Ognjenka R. Preparation of nanoemulsions by high-energy and low-energy emulsification methods. In CMBEBIH Springer; 2017. p. 317-22.

44. Wu L, Bi Y, Wu H. Formulation optimization and the absorption mechanisms of nanoemulsion in improving baicalin oral exposure. Drug Dev Ind Pharm 2017;12:1-32.

45. Gueutin C, Frebourg G, Burucoa C, Faivre V. Erythromycin encapsulation in nanoemulsion-based delivery systems for treatment of helicobacter pylori infection: protection and synergy. Biochem Biophys Res Commun 2017;493:146-51.

46. Jain N, Gupta BP, Thakur N, Jain R, Banweer J, Jain DK, et al. Phytosome: a novel drug delivery system for herbal medicine. Int J Pharm Sci Drug Res 2010;2:224-8.

47. Khan J, Alexander A, Saraf S, Saraf S. Recent advances and future prospects of phyto-phospholipid complexation technique for improving pharmacokinetic profile of plant actives. J Controlled Release 2013;68:50-60.

48. Nanavati B. Phytosome: a novel approach to enhance the bioavailability of phytoconstituent. Asian J Pharm 2017;11:S453-S461.

49. Pawar HA, Bhangale BD. Phytosome as a novel biomedicine: a microencapsulated drug delivery system. J Bioanal Biomed 2017;7:6-12.

50. Chetan K, Hatware K. Phytosomes-novel drug delivery system. Ind J Drugs 2017;5:16-36.

51. Mazumder A, Dwivedi A, Fox LT, Brummer A, Preez JL, Gerber $\mathrm{M}$, et al. In vitro skin permeation of sinigrin from its phytosomecomplex. J Pharm Pharmacol 2016;68:1577-83.

52. Abdelkader H, Longman MR, Alany RG, Pierscionek B. Phytosome-hyaluronic acid systems for ocular delivery of Lcarnosin. Int J Nanomed 2016;11:2815-27.

53. Damle M, Mallya R. Development and evaluation of a novel delivery system containing phytophospholipid complex for skin aging. AAPS PharmSciTech 2016;17:607-17.

54. Das MK, Kalita B. Design and evaluation of phyto-phospholipid complexes (Phytosomes) of rutin for transdermal application. J Appl Pharma Sci 2014;4:51-7.

55. May S, Freag Yosra SR, Elnaggar Ossama Y, Abdallah. Lyophilized phytosomal nanocarriers as platforms for enhanced diosmin delivery: optimization and ex vivo permeation. Int J Nanomed 2013;8:2385-97.

56. Telange DR, Patil AT, Pethe AM, Fegade H, Anand S, Dave VS. Formulation and characterization of an apigenin-phospholipid phytosome (APLC) for improved solubility, in vivo bioavailability, and antioxidant potential. Eur J Pharm Sci 2016;108:1-14.

57. Pathan IB, Jaware BP, Shelke S, Ambekar W. Curcumin loaded ethosomes for transdermal application: formulation, optimization, in vitro and in vivo study. J Drug Delivery Sci Technol 2018;44:49-57.

58. Khan NR, Wong TW. 5-Fluorouracil ethosomes-skin deposition and melanoma permeation synergism with microwave. Artif Cells Nanomed Biotechnol 2018;14:1-10.

59. Zhang Y, Ng W, Hu J, Mussa SS, Ge Y, Xu H. Formulation and in vitro stability evaluation of ethosomal carbomer hydrogel for transdermal vaccine delivery. Colloids Surf B 2018;163:184-91.

60. Lembo D, Donalisio M, Civra A, Argenziano M, Cavalli R. Nanomedicine formulations for the delivery of antiviral drugs: A promising solution for the treatment of viral infections. Expert opinion on drug delivery. Int J Pharm 2018;15:93-114.

61. Sharma G, Goyal H, Thakur K, Raza K, Katare OP. Novel elastic membrane vesicles (EMVs) and ethosomes-mediated effective topical delivery of aceclofenac: a new therapeutic approach for pain and inflammation. Drug Delivery 2016;23:3135-45.

62. Shelke S, Shahi S, Jalalpure S, Dhamecha D. Poloxamer 407-based intranasal thermoreversible gel of zolmitriptan-loaded nanoethosomes: formulation, optimization, evaluation and permeation studies. J Liposome Res 2016;26:313-23. 
63. Sujatha S, Sowmya G, Chaitanya M, Reddy VK, Monica M, Kumar $\mathrm{KK}$, et al. Preparation, characterization and evaluation of finasteride ethosomes. Int J Drug Delivery 2016;8:1-11.

64. Jain S, Patel N, Madan P, Lin S. Formulation and rheological evaluation of ethosome-loaded carbopol hydrogel for transdermal application. Drug Dev Ind Pharm 2015;42:1315-24.

65. Ahmed TA, Khalid M, Aljaeid BM, Fahmy UA, Abd-Allah FI. Transdermal glimepiride delivery system based on optimized ethosomalnano-vesicles: preparation, characterization, in vitro, ex vivo and clinical evaluation. Int J Pharm 2016;2:245-54.

66. Shaik MR, Sadhana C, Pragathi S, Pranithanjali KR, Pavani B, Reddy TP. Formulation and in vitro evaluation of aceclofenac ethosomes. Indo Am J Pharm 2016;2:35-41.

67. Milind P, Swati S. Development and optimization of rizatriptan benzoate ethosomes. Int J Appl Pharm 2018;10:83-90.

68. Owuor JJ, Oloo F, Ouma D, Omwoyo WN, Gathirwa JW. Optimization and characterization of primaquine-loaded solid lipid nanoparticles (SLN) for liver schinonticide targeting by freeze drying. MOJ Drug Des Delivery Ther 2017;4:21-9.

69. Bhupinder K, Newton MJ. Acyclovir solid lipid nanoparticles for skin drug delivery: fabrication, characterization and in vitro study. Recent Pat Drug Delivery 2017;11:132-46.

70. Jannin V, Blas L, Chevrier S, Miolane C, Demarne F, Spitzer D. Evaluation of the digestibility of solid lipid nanoparticles of glyceryl dibehenate produced by two techniques: ultrasonication and spray-flash evaporation. Eur J Pharm Sci 2018;111:91-5.

71. Cirri M, Mennini N, Maestrelli F, Mura P, Ghelardini C, Mannelli LD. Development and in vivo evaluation of an innovative "Hydrochlorothiazide-in cyclodextrins-in solid lipid nanoparticles" formulation with sustained release and enhanced oral bioavailability for potential hypertension treatment in pediatrics. Int J Pharm 2017;521:73-83.
72. Kotmakçı M, Akbaba H, Erel G, Ertan G, Kantarcı G. Improved method for solid lipid nanoparticle preparation based on hot microemulsions: preparation, characterization, cytotoxicity, and hemocompatibility evaluation. AAPS Pharm Sci Tech 2017;18:1355-65.

73. Thakkar A, Chenreddy S, Thio A, Khamas W, Wang J, Prabhu S. Preclinical systemic toxicity evaluation of chitosan-solid lipid nanoparticle-encapsulated aspirin and curcumin in combination with free sulforaphane in BALB/c mice. Int J Nanomed 2016;11:3265-76.

74. Chantaburanan T, Teeranachaideekul V, Chantasart D, Jintapattanakit A, Junyaprasert VB. Effect of binary solid lipid matrix of wax and triglyceride on lipid crystallinity, drug-lipid interaction and drug release of ibuprofen-loaded solid lipid nanoparticles (SLN) for dermal delivery. J Colloid Interface Sci 2017;504:247-56.

75. Wang T, Hu Q, Zhou M, Xue J, Luo Y. Preparation of ultra-fine powders from polysaccharide-coated solid lipid nanoparticles and nanostructured lipid carriers by innovative nanospray drying technology. Int J Pharm 2016;511:219-22.

76. Mosallaei N, Mahmoudi A, Ghandehari H, Yellepeddi VK, Jaafari MR, Malaekeh-Nikouei B. Solid lipid nanoparticles containing 7ethyl-10-hydroxycamptothecin (SN38): preparation, characterization, in vitro, and in vivo evaluations. Eur J Pharm Sci 2016;104:42-50.

77. Chetoni P, Burgalassi S, Monti D, Tampucci S, Tullio V, Cuffini $\mathrm{AM}$, et al. Solid lipid nanoparticles as a promising tool for intraocular tobramycin delivery: Pharmacokinetic studies on rabbits. Eur J Pharma Biopharm 2016;109:214-23.

78. Surekha R, Sumathi T. An efficient encapsulation of thymoquinone using solid lipid nanoparticle for brain-targeted drug delivery: physicochemical characterization, pharmacokinetics and biodistribution studies. Indian J Pharm Clin Res 2016;8:1616-24. 